\title{
Violência psicológica e contexto familiar de adolescentes usuários de serviços ambulatoriais em um hospital pediátrico público terciário
}

\author{
Psychological violence and the family context of adolescent users \\ of outpatient services in a public tertiary pediatric hospital
}

Cecy Dunshee de Abranches ${ }^{1}$

Simone Gonçalves de Assis ${ }^{2}$

Thiago de Oliveira Pires ${ }^{2}$

${ }^{1}$ Instituto Nacional de Saúde da Mulher, da Criança e do Adolescente Fernandes. Av. Rui Barbosa $716 / 2^{\circ}$ prédio dos fundos, Flamengo. 22.250-020 Rio de Janeiro RJ. cecy@iff.fiocruz.br ${ }^{2}$ Escola de Saúde Pública Sérgio Arouca, Claves, Fiocruz.
Abstract The scope of this research was to investigate the association of psychological violence $(P V)$ during adolescence with social and demographic factors, family structure/relationships and with other forms of maltreatment. A questionnaire was applied to 229 adolescents (11-18 years) in outpatient services in a state-run tertiary pediatric hospital. It was found that $26.4 \%$ of respondents suffered severe $P V$ in the family context, and only 5 of the respondents reported they did not suffer any $P V$ in the family context, illustrating how this kind of violence is seen as commonplace in the family relationship. The most common forms of $P V$ behavior were: being criticized for what one does or says; not being encouraged when trying to act autonomously; being called nasty names, and having an adult saying one is wrong when one tries to act. Dissatisfaction of parents with the adolescent, the nuclear family structure, the position of the child among siblings sharing the same parents were associated with PV occurring within the family context. In order to enable it to detect signs of $P V$, the health sector can promote the right to comprehensive health of adolescents, confirming itself as one of the main social sectors capable of acting preventively on the forms of violence suffered and practiced by the family group.

Key words Psychological violence, Adolescent, Family, Outpatient service
Resumo Buscou-se investigar a associação da violência psicológica (VP) na adolescência com fatores sociodemográficos, estrutura/relacionamento familiar e com outras formas de maus-tratos. Aplicou-se em 229 adolescentes (11-18 anos) um questionário, em serviços ambulatoriais de um hospital pediátrico público terciário. Encontrou-se que $26,4 \%$ dos entrevistados sofrem VP severa no contexto familiar, sendo que apenas cinco relataram não sofrer qualquer tipo de VP familiar, sugerindo que essa violência é considerada como uma forma corriqueira de relacionamento familiar. Comportamentos de VP familiares mais relatados: ser criticado pelo que faz ou diz, não ser encorajado quando tenta atuar de forma autônoma, ser chamado por nomes desagradáveis e ter adulto dizendo que está errado ao tentar agir. Insatisfação dos responsáveis com o adolescente, a estrutura familiar nuclear, a posição da criança entre os irmãos e o compartilhamento dos mesmos pais pelos irmãos mostraram-se associados à VP que ocorre no contexto familiar. O setor saúde, capacitando-se para detectar os sinais de VP, pode promover o direito à saúde integral de adolescentes, confirmando ser um dos principais setores sociais capazes de atuar preventivamente sobre as formas de violência sofridas e praticadas pelo grupo familiar.

Palavras-chave Violência psicológica, Adolescente, Família, Serviço ambulatorial 


\section{Introdução}

O fenômeno da violência, atingindo parcela importante da população e repercutindo significativamente sobre a saúde das pessoas, configurou-se como um problema de Saúde Pública relevante, tornando-se um desafio para os gestores do Sistema Unificado de Saúde (SUS) ${ }^{1-3}$. Além da magnitude da mortalidade, a morbidade das vítimas de violência delegou ao sistema de saúde os desafios de construir e consolidar outras formas de abordagem na atenção, prevenção e promoção de saúde ${ }^{3}$.

Diferentes formas da violência existentes no país têm impacto direto sobre as famílias. Estas podem tanto exercer cuidado e proteção sobre a prole como podem ser agentes da violência. $\mathrm{O}$ domicílio é local privilegiado para a ocorrência de grande parte dos eventos violentos que acometem crianças e adolescentes ${ }^{4,5}$. Os agravos decorrentes dependem da idade da criança/adolescente, da frequência com que ocorre o tipo de abuso, da relação de proximidade que ela tem com o autor da agressão e das consequências dessa situação na vida ${ }^{6}$. O setor saúde, por ter um papel privilegiado junto ao grupo familiar, se constitui em um dos setores sociais capazes de atuar preventivamente sobre as formas de violência sofridas e praticadas no ambiente familiar ${ }^{7}$.

Pesquisas científicas indicam prejuízos à saúde e ao crescimento e desenvolvimento de crianças e adolescentes, a curto e longo prazo, quando há a exposição à violência ${ }^{1,8-12}$. Sendo essas faixas etárias, períodos muito relevantes para a maturação do cérebro, as experiências no começo da vida exercem influência em padrões de comportamentos da vida adulta. Estudos comprovam que a experiência de estresse emocional precoce, inclusive os maus-tratos, levam a déficits em funções neurocognitivas, como atenção, inteligência, linguagem, funções executivas e tomada de decisões, assim como alterações no hipocampo, corpo caloso, córtex pré-frontal e córtex cingulado anterior ${ }^{13,14}$ e podem alterar a função do eixo hipotálamo-pituitária-adrenal ${ }^{15}$, que tem sido associada com as desordens depressivas e a reação de tropismo do hormônio cortisol, assim como o comportamento do indivíduo ${ }^{16}$.

Pesquisas investigam a associação do aumento da secreção do cortisol com a exposição à violência na infância, como: estudo longitudinal com 236 crianças (idade média de 7,6 anos) encontrou a associação dos maus-tratos infantis com as funções sociais (comportamento disruptivo e agressivo) e altos níveis de cortisol, após um ano de investigação ${ }^{17}$; outro estudo encontrou o aumento da reação ao cortisol para estresse psicológico em adolescentes com depressão severa com história de maus-tratos na infância ${ }^{18}$.

Uma das formas de maus-tratos contra crianças e adolescentes de difícil detecção, porém muito lesiva para a formação do indivíduo, é a violência psicológica (VP). Esta ocorre, quando os adultos sistematicamente humilham, demonstram falta de interesse, tecem críticas excessivas, induzem culpa, desencorajam, ignoram sentimentos ou cobram excessivamente a criança ou o adolescente ${ }^{19}$.

Diferentes ângulos têm sido adotados pelos autores ao estudarem VP na infância. Uns focalizam a conceituação no comportamento dos pais, em que estes repetidamente convencem a criança de que ela é a pior, não amada, não querida, ou que seu único valor é comparado com a necessidade dos outros ${ }^{20,21}$. Outros sinalizam a VP como ponto central do abuso infantil e da negligência $^{22}$, e podendo causar mais danos no desenvolvimento infantil do que a violência física ${ }^{23}$.

A visibilidade da VP na infância e adolescência no contexto familiar vem aumentando na última década, especialmente através de estudos epidemiológicos ${ }^{24}$, como a ocorrência de 10-20\% de VP severa em crianças e $50 \%$ em adolescentes, em estudo com uma amostra representativa nacional com 991 pais americanos ${ }^{25}$. Outros estudos mostram prevalências diferenciadas: $50 \% \mathrm{em}$ amostra de 217 estudantes (14-18 anos) do sexo feminino, que vivem em Bedouin-Arab, Israel ${ }^{26}$; $48 \%$ em amostra de 1.685 estudantes (11-19 anos) de escolas públicas e privadas do Município de São Gonçalo (RJ) ${ }^{27} ; 29 \%$ em amostra de 1.293 crianças/adolescentes (0-17 anos) registradas em Conselhos Tutelares de Feira de Santana (BA) em 2003 e $2004^{28} ; 94,8 \%$ em amostra de 524 acompanhantes de crianças (1-12 anos) em dois hospitais de emergência no Município do Rio de Janeiro (RJ $)^{29}$ e 96,7\% em amostra sistemática de 278 crianças adscritas ao Programa Médico de Família de Niterói (RJ) ${ }^{30}$.

Estudo com 668 mulheres pacientes de uma clínica ginecológica americana, com o objetivo de conhecer os efeitos dos maus-tratos infantis sobre a vida adulta, encontrou que o abuso mais relatado foi a VP $(37,4 \%)^{31}$; em outro estudo com amostra de 205 mulheres em serviço de atenção básica de saúde americana encontrou a VP sofrida na infância associada com problemas físicos e psicológicos (ansiedade, depressão, estresse póstraumático, ideação suicida ${ }^{32}$. A VP está associada também à disfunção de personalidade no 
adulto jovem ${ }^{10}$ e com baixa competência socioemocional na adolescência9 .

Devido aos agravos que a exposição à VP tem sobre o crescimento e o desenvolvimento de adolescentes e da escassez de estudos que retratem este tema em ambulatórios de serviços de saúde, buscou-se neste artigo estimar a prevalência da VP e avaliar a associação com fatores sociodemográficos, estrutura e relacionamento familiar e com vitimização por formas de violência física e sexual em usuários de serviços ambulatoriais de um hospital pediátrico público terciário.

\section{Metodologia}

$\mathrm{O}$ artigo se baseia em inquérito epidemiológico realizado entre janeiro e setembro de 2011, com amostra de 229 usuários de 11 a 18 anos e 221 responsáveis destes, num hospital pediátrico público terciário, localizado no Município do Rio de Janeiro (RJ). Este é um hospital referência para o SUS na saúde de crianças, adolescentes e mulheres, tendo sua demanda referida de todas as áreas programáticas do município do Rio de Janeiro, assim como de outros municípios e estados.

Um total de 229 adolescentes de 11 a 18 anos foi entrevistado na pesquisa, sendo o tamanho de amostra obtido condizente com o atendimento oferecido a pacientes da faixa etária de 11-18 anos nos três ambulatórios do hospital. A faixa etária investigada foi decorrente da escolha da escala YRS (Youth Self-Report) ${ }^{33}$, cuja aplicabilidade é em adolescentes de 11 a 18 anos.

Os critérios de inclusão dos sujeitos de pesquisa foram: adolescente de ambos os sexos, na faixa etária citada, com capacidade intelectual para responder e compreender as perguntas do questionário, acompanhados por responsáveis que convivam com os mesmos durante o último ano e que possam fornecer informações sobre o adolescente; os adolescentes de 18 anos completos podiam estar desacompanhados.

Através de entrevistas presenciais, as informações de interesse foram colhidas pela pesquisadora principal e por duas assistentes previamente treinadas. As entrevistas foram realizadas nas salas de espera dos ambulatórios selecionados, enquanto os usuários aguardavam a consulta agendada.

Realizou-se o inquérito epidemiológico através de dois questionários constituídos por escalas selecionadas (com validade e confiabilidade reconhecidas), que permitiram investigar os conceitos de interesse para o estudo, sendo um ques- tionário para os responsáveis e outro para as crianças/adolescentes. O questionário dos adolescentes foi composto por perguntas sobre escolaridade, raça e pelas escalas de: $\mathrm{VP}^{34}$, resiliência ${ }^{35,36}$ e $\mathrm{YRS}^{33}$ (que estima a presença de problemas de saúde mental).

O instrumento dos responsáveis foi composto por perguntas sobre: variáveis sociodemográficas; relacionamento familiar e envolvimento familiar com o adolescente atendido no serviço; e a escala Self-Reported Questionnaire/SRQ-20 $0^{37}$, que é um instrumento que permite avaliar a suspeição de transtornos psiquiátricos menores, mas não a detecção de distúrbios psiquiátricos específicos ou níveis de intensidade.

As principais variáveis e medidas utilizadas foram:

a) Os fatores sociodemográficos relevantes ao estudo (sexo, raça, faixa etária, escolaridade do entrevistado e de seus responsáveis);

b) Nível socioeconômico das famílias: segue o critério de classificação econômica da Associação Brasileira de Empresas de Pesquisa (ABEP). Estimador da capacidade de consumo, a partir de indicadores, como: televisão a cores, banheiro, automóvel, empregada mensalista, geladeira duplex ou freezer e grau de instrução do chefe da família. Estratos A, B e C correspondem às camadas alta e média; e D e E às camadas populares.

c) O ambiente de convivência familiar do entrevistado (estrutura familiar com pais juntos, separados, pais adotivos, sem ambos os pais ou família monoparental; relacionamento familiar com pai, mãe e irmãos, diálogo familiar e respeito na família; convivência, compreensão e aceitação familiar);

Funcionamento geral da família, avaliado pela General Functioning Scale of the McMaster Family Assessment Device ${ }^{38}$, que inclui 12 perguntas: é difícil planejar atividades familiares, porque há desentendimentos; em tempos de crise, pode-se buscar ajuda uns nos outros; não se pode conversar na família sobre a tristeza que sentem; cada pessoa é aceita pelo que é; evita-se discutir medos ou preocupações; mostram-se sentimentos uns pelos outros; sentimentos ruins na família; sentir-se aceito pelo que é; dificuldade em tomar decisões em família; ser capaz de tomar decisões; não se dar bem juntos; confiar uns nos outros. As opções de respostas oscilam de: concordo totalmente a discordo totalmente (1-5 pontos), com valores maiores, significando melhor funcionamento geral da família. Neste artigo avalia-se o precário funcionamento familiar por resultados na faixa de um desvio padrão abaixo da média e 
o funcionamento regular ou bom acima deste nível.

d) Envolvimento parental: a ausência (crianças com resultados caindo na faixa de um desvio padrão abaixo da média) e a presença de envolvimento parental, aferido pelas seguintes perguntas: com que frequência o responsável elogia a criança, dizendo coisas, como: "Muito bom!" ou "Que coisa legal você fez!" ou "Isso está indo bem"!; com que frequência o responsável e a criança conversam ou brincam um com outro, focando atenção um no outro por cinco minutos ou mais, apenas por diversão?; com que frequência o responsável e a criança riem juntos?; com que frequência o responsável faz alguma coisa especial com a criança, que ela gosta?; com que frequência o responsável pratica esportes, hobbies ou jogos com a criança?; com que frequência o responsável lê com a criança?. São quatro opções de respostas, valendo de 1 a 4 pontos: sempre, muitas vezes, poucas vezes e nunca. Os escores maiores do que o desvio padrão abaixo da média significam a existência de envolvimento do responsável com a criança.

e) A escala Self-Reported Questionnaire/SRQ$20^{37}$, que é um instrumento que permite avaliar a suspeição de transtornos psiquiátricos menores, mas não a detecção de distúrbios psiquiátricos específicos ou níveis de intensidade, é composta por 20 itens (sim/não), englobando sintomas psicossomáticos, sintomas depressivos e sintomas de ansiedade, onde a presença de 8 ou mais sintomas para as mulheres e 7 para os homens caracterizaria a existência do transtorno.

Como variável de exposição, investigou-se o sofrer VP de pessoas da família, que é mensurada através da escala específica ${ }^{39} \mathrm{e}$ que avalia as experiências vividas por jovens que foram diminuídos em suas qualidades, capacidades, desejos, emoções e também se foram excessivamente cobrados por pessoas significativas. É constituída por 18 itens com opções de respostas que variam do nunca até o sempre, sendo adaptada transculturalmente à realidade brasileira ${ }^{34}$, apresentando as propriedades psicométricas de $\alpha$ de Cronbach de 0,91 e Coeficiente de Correlação Intraclasse (ICC) de 0,8629 . Os resultados obtidos na avaliação da validade de constructo pelas autoras mostraram evidências da adequação da versão proposta. Imediatamente após a aplicação da escala foi indagado ao adolescente quais pessoas significativas mais agiram com VP sobre o adolescente: pai, mãe, madrasta, padrasto, irmãos, tios, avós e outros.

$\mathrm{O}$ escore da escala de VP foi definido como a razão entre o somatório dos pontos associados às frequências de cada um dos itens (de 1 - nunca a 5 - sempre) e a pontuação máxima que seria alcançada (90) multiplicada por 100 . O ponto de corte foi estabelecido como valores acima e abaixo do terceiro quartil, sendo categorizadas como ausente a moderada (que inclui adolescentes que respondem nunca, às vezes ou raramente na maioria dos itens) e severa (frequências elevadas na maioria das respostas - sempre/quase sempre).

A análise dos dados foi composta por frequências simples de cada uma das variáveis que compõem o banco. Foram utilizadas tabelas de contingência e testes de associação (qui-quadrado de Pearson e teste exato de Fisher para tabela 2x2 com nível de significância de 5\%), para se verificar a relação entre VP e outras variáveis, que, segundo a literatura, haveria algumas possíveis ligações. Medidas de associação (razão de chances) também foram utilizadas com intervalos de confiança de $95 \%$.

Este estudo observou as diretrizes da Resolução 196/9640 do Conselho Nacional de Saúde e os princípios da Declaração de Helsinki aprovados pelo Comitê de Ética em Pesquisa do hospital, local onde esta ocorreu. As entrevistas foram realizadas mediante a assinatura do Termo de Consentimento Livre e Esclarecido pelo responsável pelo adolescente.

\section{Resultados}

A amostra foi composta por 229 adolescentes de 11 a 18 anos, com idade média de 13,5 anos (desvio padrão $=2,0$ ), sendo $53,2 \%$ do sexo masculino. A cor da pele/raça autorreferida pelos entrevistados foi: branca $33,8 \%$, preta $19,5 \%$, parda $42,4 \%$ e amarelo/indígena $4,3 \%$. Um total de $83,5 \%$ dos adolescentes pertence aos estratos sociais populares (C e D). Encontrou-se que 14,3\% dos entrevistados apresentaram uma defasagem na escolaridade de dois ou mais anos (relação entre idade cronológica e série declarada).

De acordo com o critério adotado para definir VP severa, tem-se que 61 crianças/adolescentes $(26,4 \%)$ se enquadram neste quadro. Este achado indica que a VP é uma forma de comunicação familiar corriqueira (um em cada quatro adolescentes assim vivem.

Outro aspecto que reitera este achado é que apenas $5(2,2 \%)$ adolescentes responderam nunca aos 18 itens de VP indagados na pesquisa. Estes cinco casos foram incluídos na categoria VP ausente a moderada, que é essencialmente composta pelos que a sofrem em frequência menor, 
na maioria dos itens da escala (entre 93,5\% e $100 \%$ ).

Antes de apresentar os cruzamentos que avaliam a associação da VP com atributos sociodemográficos, familiares e com outras vivências de violência, vale a pena atentar para a Tabela 1 , que mostra como os diferentes itens da escala de VP se distribuem na amostra investigada. Os comportamentos reiteradamente presentes no relacionamento (frequência sempre/quase sempre), que foram apontados por mais de 10\% dos adolescentes, são: ser criticado pelo que faz ou diz; não ser encorajado quando tenta atuar de forma autônoma; ser chamado por nomes desagradáveis e ter adulto dizendo que está errado ao tentar agir. Alguns atos que compõem o relacionamento familiar abusivo, relatados com menor frequência (às vezes/raramente), são: culpabilização por falhas $(58,5 \%)$; críticas das coisas que o entrevistado faz e diz (58\%); gritos sem motivo $(45,4 \%)$; não acreditar no entrevistado (53,7\%) e dizer ao entrevistado que ele está errado quando diz e faz alguma coisa $(45,4 \%)$.

No relato dos entrevistados sobre quem mais age com VP na família, pode-se verificar no Gráfico 1, que a mãe é a principal agente desta forma de violência seguida pelo pai e pelos irmãos. $\mathrm{Na}$ categoria outros estão primos e sobrinhos, além de outras pessoas, fora do circuito familiar, como: amigos, vizinhos, namorados, professores e uma diretora da escola, também lembrados por praticar VP sobre o adolescente.

Vale apontar que, se levada em conta a ordem de prioridade na fala dos jovens, os mais lembrados em primeiro lugar são a mãe, o padrasto e os tios, só depois vindo o pai e os irmãos. Interessante ressaltar que apenas 9,3\% dos adolescentes vivem com padrasto e, no entanto, $6,9 \%$ do total de entrevistados se queixam do abuso emocional cometido por esta figura de autoridade.

$\mathrm{Na}$ Tabela 2, a seguir, pode-se constatar que não há diferença estatisticamente significativa quanto à vivência de VP, segundo o sexo e quanto à renda familiar. No que se refere à cor da pele/ raça, tem que os adolescentes que se referem pardos têm duas vezes a chance de sofrer VP severa, por parte de pessoas de sua família, do que os que se afirmam brancos.

$\mathrm{Na}$ associação com a estrutura familiar, na Tabela 3, observa-se que ter irmãos de diferentes casamentos eleva a chance de sofrer VP severa $(\mathrm{OR}=3,09)$, se comparado aos entrevistados com irmãos do mesmo casamento dos pais. Ser filho do meio mostra mais chance de sofrer VP severa $(\mathrm{OR}=3,46)$ do que as outras posições na

Tabela 1. Itens de violência psicológica $(\mathrm{N}=229)$.

\begin{tabular}{lccc}
\hline \multicolumn{1}{c}{ Itens } & $\begin{array}{c}\text { Sempre/ } \\
\text { Quase sempre }\end{array}$ & $\begin{array}{c}\text { Às vezes/ } \\
\text { raramente }\end{array}$ & Nunca \\
\hline Humilhou o adolescente & 3,0 & 42,8 & 54,1 \\
Demonstrou falta de interesse pelo adolescente & 2,6 & 44,6 & 52,8 \\
Culpou o adolescente por falhas que o familiar cometeu & 11,3 & 58,5 & 30,3 \\
Culpou o adolescente por falhas cometidas por outras pessoas & 6,9 & 44,2 & 48,9 \\
Criticou o adolescente pelas coisas que faz e diz & 14,3 & 58,0 & 27,7 \\
Criticou o adolescente pela aparência e pelo modo como se veste & 8,7 & 30,7 & 60,6 \\
Criticou os amigos do adolescente & 10,0 & 41,9 & 48,1 \\
Não elogiou o adolescente quando este tinha feito algo de bom & 11,2 & 40,3 & 48,5 \\
Não encorajou o adolescente quando ele quis fazer algo por si mesmo & 13,0 & 32,1 & 55,0 \\
Disse ao adolescente que ele não era tão bom quanto as outras pessoas & 3,5 & 27,3 & 69,3 \\
Gritou ou berrou com o adolescente sem nenhum motivo & 10,0 & 45,4 & 44,6 \\
Chamou o adolescente de nomes desagradáveis como "doido", "idiota" & 12,1 & 40,2 & 47,6 \\
ou "burro" & & & \\
Fez piadas sobre o adolescente na frente de outras pessoas & 8,6 & 24,6 & 56,7 \\
Não acreditou no adolescente & 10,4 & 53,7 & 35,9 \\
Disse ao adolescente que estava errado quando ele disse ou fez & 12,6 & 45,4 & 42,0 \\
alguma coisa & & & \\
Ignorou o adolescente quando este procurava carinho & 6,9 & 17,3 & 75,8 \\
Forçou o adolescente a assumir responsabilidade pela maioria das & 7,8 & 30,3 & 61,9 \\
tarefas domésticas & & & 76,6 \\
Humilhou o adolescente, fazendo piadas sobre o seu corpo & 7,8 & 15,6 & 76,6 \\
\hline
\end{tabular}




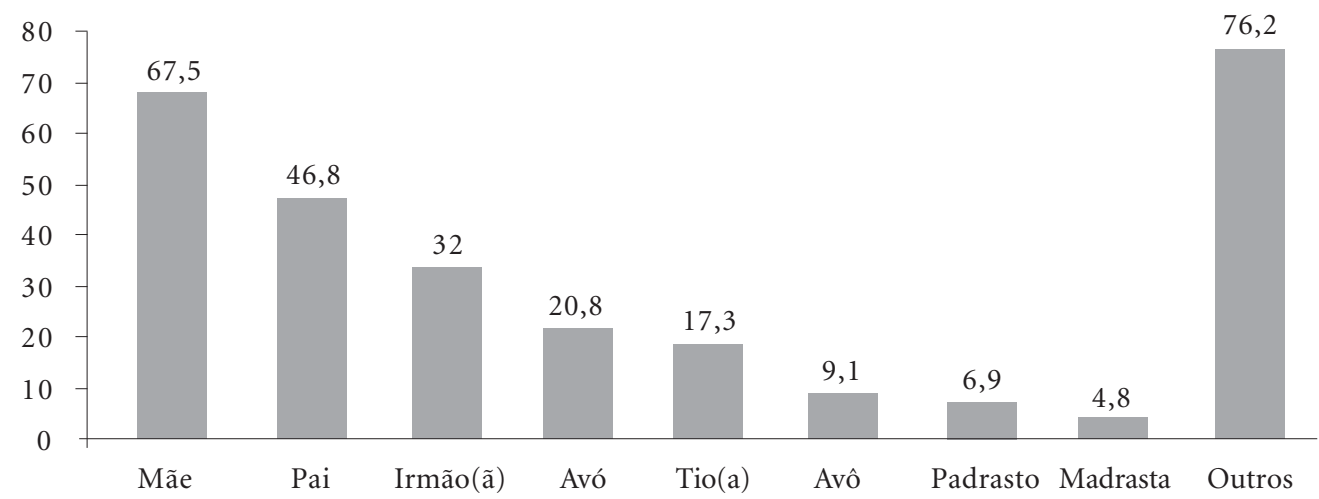

Gráfico 1. Frequência de familiares/pessoas que praticam violência psicológica.

\begin{tabular}{|c|c|c|c|c|c|c|}
\hline \multirow[t]{2}{*}{ Variáveis } & \multirow[t]{2}{*}{ Itens } & \multicolumn{2}{|c|}{ VP ausente a moderada } & \multicolumn{2}{|c|}{ VP severa } & \multirow[t]{2}{*}{ OR (IC) } \\
\hline & & $\mathbf{N}$ & $\%$ & $\mathbf{N}$ & $\%$ & \\
\hline \multirow[t]{2}{*}{ Sexo } & Masculino & 87 & 51,2 & 36 & 59,0 & $1,37(0,76-2,48)$ \\
\hline & Feminino & 83 & 48,8 & 25 & 41,0 & 1 \\
\hline \multirow[t]{4}{*}{ Cor/raça } & Preta & 33 & 19,4 & 12 & 19,7 & $1,53(0,64-3,64)$ \\
\hline & Parda & 66 & 38,8 & 32 & 52,5 & $2,04(1,01-4,12)$ \\
\hline & Amarela/indígena & 8 & 4,7 & 2 & 3,3 & $1,05(0,20-5,46)$ \\
\hline & Branca & 63 & 37,1 & 15 & 24,6 & 1 \\
\hline \multirow[t]{2}{*}{ Estrato social } & $\mathrm{C}+\mathrm{D}$ & 127 & 85,8 & 40 & 76,9 & $0,55(0,25-1,22)$ \\
\hline & $A+B$ & 21 & 14,2 & 12 & 23,1 & 1 \\
\hline \multirow[t]{4}{*}{ Renda (SM) } & $\leq 1 / 4 \mathrm{SM}$ & 15 & 9,3 & 5 & 8,3 & $0,83(0,24-2,91)$ \\
\hline & $>1 / 4$ a $1 / 2 \mathrm{SM}$ & 38 & 23,5 & 15 & 25,0 & $0,99(0,38-2,54)$ \\
\hline & $>1 / 2$ a $1 \mathrm{SM}$ & 84 & 51,9 & 30 & 50,0 & $0,89(0,38-2,08)$ \\
\hline & $>1$ & 25 & 15,4 & 10 & 16,7 & 1 \\
\hline
\end{tabular}

${ }^{*}$ Destaque para associações significativas em nível de $5 \%$

família. Ter só o pai ou só a mãe (família monoparental) indica mais chance de sofrer VP severa $(\mathrm{OR}=2,5)$ do que se observa entre aqueles que vivem com pai e mãe (família nuclear). Outra variável apresentada na Tabela 3, que mostra diferença, segundo a vitimização por VP severa, é satisfação do responsável com a criança/adolescente sob seus cuidados: aqueles que se declaram mais ou menos satisfeitos ou insatisfeitos mostram mais tendência em ter filhos que informam vítimas de VP severa na família $(\mathrm{OR}=2,5)$ do que os entrevistados que têm responsáveis plenamente satisfeitos em relação à criança/adolescente. Para as demais variáveis apresentadas na Tabela 3, não se constata diferença estatisticamente significativa quanto à vitimização por VP.
No que tange ao uso de substâncias pelos responsáveis, destaca-se o maior consumo de uso remédios para acalmar ou para dormir entre as mães e o de álcool ao ponto de embriaguez entre os pais (segundo o relato das mães/responsáveis), embora não tenha havido associação estatística entre tal consumo e o relato do adolescente sobre VP na família. Nenhuma mãe informou utilizar maconha, cocaína, crack ou pasta de coca no último ano; oito pais relataram tal consumo (segundo o relato da mãe/responsável), sem diferenciação, segundo presença de VP referida pelo adolescente (dados não apresentados).

$\mathrm{Na}$ Tabela 4, avalia-se a associação de VP na família com agressão física perpetrada por familiar no passado e na atualidade e com agressão 
Tabela 3. Associações entre variáveis do contexto familiar e violência psicológica.

\begin{tabular}{|c|c|c|c|c|c|c|}
\hline \multirow[t]{2}{*}{ Variáveis } & \multirow[t]{2}{*}{ Itens } & \multicolumn{2}{|c|}{$\begin{array}{l}\text { VP ausente a } \\
\text { moderada }\end{array}$} & \multicolumn{2}{|c|}{ VP severa } & \multirow[t]{2}{*}{ OR (IC 95\%) } \\
\hline & & $\mathbf{N}$ & $\%$ & $\mathbf{N}$ & $\%$ & \\
\hline \multirow[t]{3}{*}{ Pais } & Separados & 41 & 25,3 & 21 & 35,0 & $1,75(0,91-3,37)$ \\
\hline & Nunca viveram juntos & 8 & 4,9 & 6 & 10,0 & $2,57(0,83-7,93)$ \\
\hline & Vivem juntos & 113 & 69,8 & 33 & 55,0 & 1 \\
\hline \multirow[t]{2}{*}{ Irmãos de } & Diferentes casamentos & 39 & 27,7 & 26 & 54,2 & $3,09(1,57-6,08)^{*}$ \\
\hline & Todos dos mesmos pais & 102 & 72,3 & 22 & 45,8 & 1 \\
\hline No de crianças/ & $\geq 3$ & 101 & 61,6 & 37 & 60,7 & $0,96(0,53-1,76)$ \\
\hline adolescentes na casa & $<3$ & 63 & 38,4 & 24 & 39,3 & 1 \\
\hline \multirow[t]{4}{*}{ Posição entre irmãos } & Mais velho & 43 & 26,5 & 11 & 19,0 & $0,98(0,43-2,26)$ \\
\hline & Único & 26 & 16,0 & 10 & 17,2 & $1,48(0,61-3,59)$ \\
\hline & Meio & 20 & 12,3 & 18 & 31,0 & $3,46(1,53-7,79)$ \\
\hline & Mais novo & 73 & 45,1 & 19 & 32,8 & 1 \\
\hline \multirow[t]{4}{*}{ Estrutura familiar } & $\begin{array}{l}\text { Pai/madrasta; Mãe/ } \\
\text { padrasto }\end{array}$ & 15 & 9,3 & 6 & 10,2 & $1,42(0,51-3,94)$ \\
\hline & Só pai ou só mãe & 27 & 16,8 & 19 & 32,2 & $2,50(1,24-5,04)$ \\
\hline & Sem pai sem mãe & 2 & 1,2 & 1 & 1,7 & $1,77(0,16-20,16)$ \\
\hline & Pai e mãe & 117 & 72,7 & 33 & 55,9 & 1 \\
\hline \multirow[t]{2}{*}{ Funcionamento familiar } & Precário & 24 & 14,7 & 11 & 18,6 & $1,33(0,61-2,91)$ \\
\hline & Regular/bom & 139 & 85,3 & 48 & 81,4 & 1 \\
\hline \multirow[t]{2}{*}{ Envolvimento familiar } & Ausência & 15 & 9,3 & 11 & 18,0 & $2,16(0,92-5,00)$ \\
\hline & Presença & 147 & 90,7 & 50 & 82,0 & 1 \\
\hline $\begin{array}{l}\text { Como se sente como } \\
\text { responsável pelo }\end{array}$ & $\begin{array}{l}\text { Mais ou menos } \\
\text { satisfeito/ insatisfeito }\end{array}$ & 13 & 8,2 & 11 & 18,3 & $2,50(1,05-5,95)$ \\
\hline entrevistado & Muito satisfeito & 145 & 91,8 & 49 & 81,7 & 1 \\
\hline \multirow{2}{*}{$\begin{array}{l}\text { Sofrimento psíquico / } \\
\text { responsável }\end{array}$} & Tem problemas & 30 & 20,7 & 18 & 32,1 & $1,82(0,91-3,62)$ \\
\hline & Não tem problemas & 115 & 79,3 & 38 & 67,9 & 1 \\
\hline Escolaridade do & Não sabe ler e escrever & - & - & - & - & - \\
\hline \multirow[t]{3}{*}{ responsável feminino } & $\begin{array}{l}\text { Fundamental } \\
\text { incompleto/ completo }\end{array}$ & 52 & 31,7 & 21 & 34,4 & $0,87(0,31-2,43)$ \\
\hline & $\begin{array}{l}\text { Médio incompleto/ } \\
\text { completo }\end{array}$ & 97 & 59,1 & 33 & 54,1 & $0,73(0,27-1,94)$ \\
\hline & $\begin{array}{l}\text { Superior incompleto/ } \\
\text { completo }\end{array}$ & 15 & 9,1 & 7 & 11,5 & 1 \\
\hline Escolaridade do & Não sabe ler e escrever & 2 & 1,3 & - & - & - \\
\hline \multirow[t]{3}{*}{ responsável masculino } & $\begin{array}{l}\text { Fundamental } \\
\text { incompleto/ completo }\end{array}$ & 58 & 38,9 & 24 & 46,2 & $1,10(0,27-4,52)$ \\
\hline & $\begin{array}{l}\text { Médio incompleto/ } \\
\text { completo }\end{array}$ & 81 & 54,4 & 25 & 48,1 & $0,82(0,20-3,34)$ \\
\hline & $\begin{array}{l}\text { Superior incompleto/ } \\
\text { completo }\end{array}$ & 8 & 5,4 & 3 & 5,8 & 1 \\
\hline \multirow{4}{*}{$\begin{array}{l}\text { Mãe usou bebida alcoólica } \\
\text { até se embriagar } \\
\text { Pai usou bebida alcoólica } \\
\text { até se embriagar }\end{array}$} & Sim & 5 & 3,0 & 4 & 6,6 & $2,23(0,58-8,60)$ \\
\hline & Não & 159 & 97,0 & 57 & 93,4 & 1 \\
\hline & Sim & 24 & 15,6 & 13 & 23,6 & $1,68(0,79-3,58)$ \\
\hline & Não & 130 & 84,4 & 42 & 76,4 & 1 \\
\hline \multirow{4}{*}{$\begin{array}{l}\text { Mãe usou remédio pra } \\
\text { acalmar ou ajudar a dormir } \\
\text { Pai usou remédio pra } \\
\text { acalmar ou ajudar a dormir }\end{array}$} & $\operatorname{Sim}$ & 30 & 18,3 & 13 & 21,3 & $1,21(0,58-2,51)$ \\
\hline & Não & 134 & 81,7 & 48 & 78,7 & 1 \\
\hline & Sim & 7 & 4,7 & 1 & 1,9 & $0,39(0,05-3,21)$ \\
\hline & Não & 143 & 95,3 & 53 & 98,1 & 1 \\
\hline
\end{tabular}

* Destaque para associações significativas em nível de 5\%

sexual sem especificação do agressor. Encontrouse que o adolescente que sempre/muitas vezes sofreu agressão física no passado tem 5,17 vezes a chance de sofrer VP severa, em relação aos que nunca foram agredidos de tal forma. Igual cenário ocorre em relação à agressão física atual, com 
Tabela 4. Associação entre vivência de agressão física (passada e atual) perpetrada por familiar e agressão sexual com violência psicológica no contexto familiar.

\begin{tabular}{|c|c|c|c|c|c|c|}
\hline \multirow[t]{2}{*}{ Variáveis } & \multirow[t]{2}{*}{ Itens } & \multicolumn{2}{|c|}{$\begin{array}{l}\text { VP ausente } \\
\text { a moderada }\end{array}$} & \multicolumn{2}{|c|}{ VP severa } & \multirow[t]{2}{*}{ OR (IC) } \\
\hline & & $\mathbf{N}$ & $\%$ & $\mathbf{N}$ & $\%$ & \\
\hline \multirow{3}{*}{$\begin{array}{l}\text { Agressão } \\
\text { física passada }\end{array}$} & Sempre/muitas vezes* & 5 & 2,9 & 7 & 11,5 & $5,17(1,50-17,82)$ \\
\hline & Algumas vezes & 80 & 47,1 & 31 & 50,8 & $1,43(0,77-2,66)$ \\
\hline & Nunca & 85 & 50,0 & 23 & 37,7 & 1 \\
\hline \multirow{4}{*}{$\begin{array}{l}\text { Agressão física } \\
\text { atual } \\
\text { Agressão } \\
\text { sexual }\end{array}$} & Sempre/muitas/algumas vezes ${ }^{*}$ & 22 & 12,9 & 17 & 27,9 & $2,60(1,27-5,32)$ \\
\hline & Nunca & 148 & 87,1 & 44 & 72,1 & 1 \\
\hline & Sim & 1 & 0,6 & 1 & 1,6 & $2,82(0,17-45,74)$ \\
\hline & Não & 169 & 99,4 & 60 & 98,4 & 1 \\
\hline
\end{tabular}

${ }^{*}$ Destaque para associações significativas em nível de 5\%

VP severa 2,6 vezes mais frequente do que ocorre entre aqueles que nunca sofrem agressão física no momento presente. Estes dados mostram haver redução de agressão física à medida que os entrevistados crescem. Não se observou diferenciação entre sofrer agressão sexual e VP na família, valendo destacar que esta forma de agressão foi relatada por dois adolescentes de ambos os sexos (12-14 anos), envolvendo amigo do irmão e pessoa sem roupa em carro.

\section{Discussão}

A violência resulta em altos custos econômicos e sociais para a sociedade, com efeitos emocionais nas famílias, impacto na saúde, na qualidade de vida e nos anos potenciais perdidos ${ }^{41}$, indicando a relevância de investigações que objetivem a sua prevenção e a promoção de saúde.

O hospital, campo desta pesquisa, por ser referência para o SUS na saúde de crianças, adolescentes e mulheres, tem usuários de toda a região metropolitana do RJ, como também de outros municípios desse Estado. A maior parte da população investigada pertence aos estratos sociais menos privilegiados $\mathrm{C}$ e D (segundo ABEP Associação Brasileira de Empresas de Pesquisa), com renda mensal familiar entre $1 / 4$ e 1 salário mínimo e responsáveis com ensino médio incompleto/completo. A clientela que habitualmente procura os hospitais públicos do SUS ${ }^{29,30}$ configura um perfil predominante de clientela de estratos sociais C e D. Assim, pode-se pensar que, apesar de que os maus-tratos contra crianças e adolescentes possam ocorrer em qualquer classe social, a violência estrutural que afeta muitas fa- mílias brasileiras ${ }^{8}$ é um fator que agrava a capacidade de cuidar.

Estudo sobre o perfil dos atendimentos em serviços de emergência dos casos de violência contra crianças e adolescentes, entre 2006 e 2007, no Brasil ${ }^{4}$ apontou que as maiores diferenças entre meninos e meninas foram observadas nos atendimentos, cujas vítimas tinham de 5 a 9 anos, possuíam pele negra (estando a cor de pele parda incluída) e mais frequentemente do sexo feminino. No presente artigo, notou-se predomínio do sexo masculino, principalmente em relação à VP severa, porém, corroborando em relação à cor da pele/raça autorreferida pelos entrevistados, associada com VP.

No contexto familiar, a maioria dos entrevistados morava com seus pais, e a ocorrência de VP severa evidencia elevada frequência $(26,4 \%)$ de alguns atos com potencial de causar danos ao desenvolvimento infanto-juvenil, como culpabilizar, criticar, gritar, desacreditar e desmerecer. A predominância da mãe na perpetração desta forma de violência encontra respaldo na literatu$\mathrm{ra}^{30}$, bem como é relevante a presença de pai, padrasto, irmão e tio(a). A utilização de tais comportamentos no cotidiano familiar demonstra a importância de aumentar a identificação desse abuso, tirando a VP da invisibilidade, tanto por quem sofre como por quem perpetra.

A saúde é um dos principais setores sociais capazes de atuar preventivamente sobre as formas de violência sofridas e praticadas pelo grupo familiar ${ }^{7}$. Neste artigo, apenas a insatisfação dos responsáveis com o adolescente, a estrutura familiar nuclear, a posição da criança entre os irmãos e o compartilhamento dos mesmos pais pelos irmãos mostrou-se associada à VP que 
ocorre no contexto familiar. Estudos corroboram alguns dos achados como: a) a necessidade de mais atenção nas brigas entre irmãos, tão naturalizada e banalizada em nossa cultura, mas que é um sinal de disfunção parental ou fami$\operatorname{liar}^{42-44}$ b) na estrutura familiar nuclear, testemunhar violência de parceiros íntimos ${ }^{22}$ é um tipo frequente de VP; também a satisfação no casamento $^{26}$ é considerada como um fator de risco para VP. A posição entre os irmãos na família, a predisposição para ser vítima de VP, o funcionamento e o envolvimento familiar não encontram respaldo na literatura, podendo ser objeto de investigações futuras. Pesquisas sobre as interações familiares são importantes de serem realizadas, considerando os recursos a serem promovidos sob o enfoque da resiliência ${ }^{45}$.

Estes aspectos da dinâmica familiar podem ser detectados pelo profissional de saúde e pode servir como um sinal de alerta para a prevenção das relações familiares em situação de violência. Estudo sobre violência familiar e intervenção do Programa Saúde da Família de Niterói (RJ) relata que a agressão psicológica que ocorre nos lares das famílias estudadas é extremamente comum, banalizada e pouco valorizada, tanto pela sociedade que a produz quanto pelos profissionais de saúde ${ }^{30}$. Comumente não demanda notificação ou outras formas de intervenção. É salientada a necessidade de aumento do diálogo entre profissionais de saúde e usuários, atividades de educação em saúde e visitas domiciliares que abordem as questões cotidianas das famílias, constituindo-se em porta de entrada para a discussão do tema e para a mudança de atitude, ressaltando a riqueza do vínculo família/equipe de saúde que não deve ser desperdiçada.

Outro estudo ${ }^{46}$ com 67 famílias em Montreal (Canadá) com história de maus-tratos às crianças corrobora a eficácia das intervenções baseadas no apego. Durante oito semanas as famílias foram visitadas em suas casas e sensibilizadas sobre regulação do apego e da emoção, e com vídeos sobre as interações entre pais e filhos. Depois das intervenções percebeu-se aumento da sensibilidade dos pais, mais segurança no apego e redução na desorganização das crianças.

No que se refere à associação observada entre VP e agressão física (no passado e no presente) na família, se verificou a elevada frequência desta forma de agir na família, bem como sua redução à medida que o adolescente se desenvolve. A maior presença da violência física dentre os que sofrem VP indica a confluência existente entre as diferentes formas de violência. Este dado reforça a necessidade que a nossa sociedade tem em reavaliar medidas educativas com uso da força, ainda tão banalizadas ${ }^{45}$. A associação das diferentes formas de violência, evidenciadas em diversos estudos ${ }^{6,7,11,23,29}$ demonstra como é relevante a detecção pelos profissionais de saúde dos conflitos existentes na família e das estratégias "educativas" por elas adotadas.

Limitações do estudo: o tamanho da amostra que dificulta encontrar associações estatísticas, obrigando a agregar categorias de muitas variáveis que seriam melhor estudadas desagregadas; a não existência de um ponto de corte para a escala de VP com comprovada sensibilidade e especificidade $^{44}$, que dificulta a avaliação de prevalência de VP entre estudos nacionais e internacionais. Por ser um hospital de referência, pode apresentar uma população específica, com dificuldades especiais onde a ocorrência da VP seja diferenciada. Embora a aceitabilidade da pesquisa tenha sido muito boa e os procedimentos de coleta adotados terem garantido a privacidade das informações prestadas, outra provável limitação do estudo diz respeito à exigência legal da assinatura dos pais ou responsáveis do Termo de Consentimento Livre e Esclarecido, para a abordagem de adolescentes em pesquisas. $\mathrm{O}$ fato de os mesmos representarem os principais perpetradores, confirmado nesta investigação e apontado na maioria dos estudos sobre maus-tratos infantis, pode impedir ou dificultar a participação e a autonomia de adolescentes referirem abusos durante as entrevistas (nem sempre com o grau de privacidade ideal), revelando magnitude da prevalência do evento menor do que aquela que ocorre na realidade.

\section{Conclusão}

Para finalizar, dois aspectos são ressaltados em função de sua extrema relevância no enfrentamento da VP familiar. Um diz respeito à magnitude $\mathrm{e}$ aos impactos da VP na saúde de indivíduos em pleno processo de desenvolvimento, o que exige o desenvolvimento de um trabalho em rede de instituições com este público, pois a complexidade da abordagem dos casos de VP contra os adolescentes requer um cuidado multidisciplinar com abordagens individuais, familiares e comunitárias.

Outro aspecto se refere à redução da subnotificação de VP pelos serviços de saúde, pois a identificação, a sensibilização, a capacitação e a intervenção realizada pelos profissionais de saúde coloca a notificação como um instrumento importante de proteção e não de denúncia e punição. 
Assim, a identificação e a prevenção da violência contra adolescente é de fundamental importância para determinar o seu rompimento e para impedir a (re)produção do ciclo da violência intrafamiliar.

\section{Colaboradores}

CD Abranches participou da redação, da concepção e do delineamento e interpretação dos dados do artigo; SG Assis participou da elaboração, da revisão crítica e interpretação dos dados do artigo; TO Pires participou na análise dos dados do artigo.

\section{Referências}

1. Krug EG, Dahlberg LL, Mercy JA, Zwi AB, Lozano R. Relatório Mundial sobre violência e saúde. Genebra: Organização Mundial da Saúde; 2002.

2. Brasil. Ministério da Saúde (MS). Secretaria de Políticas de Saúde. Violência Familiar: orientação para prática em serviço. Brasília: Secretaria de Políticas de Saúde, MS; 2001.

3. Njaine K, Assis SG, Constantino P, organizadoras. Impactos da violência na saúde. Rio de Janeiro: Fundação Oswaldo Cruz; 2009.

4. Mascarenhas MDM, Silva MMA, Malta DC, Lima CM, Carvalho MGO, Oliveira VLA. Violência contra a criança: revelando o perfil dos atendimentos em serviços de emergência, Brasil, 2006 e 2007. Cad Saude Publica 2010; 26(2):347-357.

5. Minayo MCS, Lima CA. Processo de Formulação e ética de ação da Política Nacional de Redução de Morbimortalidade por Acidentes e Violências. In: Njaine K, Assis SG, Constantino P, organizadoras. Impactos da violência na saúde. Rio de Janeiro: Fundação Oswaldo Cruz; 2009. p. 43-56.

6. Ferreira AL, Moura ATMS, Morgado R, Gryner S, Branco VMC. Crianças e adolescentes em situação de violência. In: Njaine K, Assis SG, Constantino P, organizadoras. Impactos da violência na saúde. Rio de Janeiro: Fundação Oswaldo Cruz; 2009. p. 127148.

7. Cavalcanti FG, Schenker M. Violência, família e sociedade. In: Njaine K, Assis SG, Constantino P, organizadoras. Impactos da violência na saúde. Rio de Janeiro: Fundação Oswaldo Cruz; 2009. p. 57-77.

8. Brasil. Ministério da Saúde (MS). Impacto da violência na saúde dos brasileiros. Brasília: MS; 2005.

9. Shaffer A, Yates TM, Eehand R. The relation of emotional maltreatment to early adolescent competence: developmental processes in a prospective study. Child Abuse Negl 2009; 33(1):36-44.

10. Allen B. An analysis of the impact of diverse forms of childhood psychological maltreatment on emotional adjustment in early adulthood. Child Maltreat 2008; 13(3):307-312. 
11. Greenfield EA, Marks NF. Profiles of physical and psychological violence in childhood as a risk factor for poorer adult health: evidence from the 19952005 National Survey of Midlife in the United States. J Aging Health 2009; 21(7):943-966.

12. Tietjen GE, Brandes JL, Peterlin BL, Eloff A, Dafer RM, Stein MR, Drexler E, Martin VT, Hutchinson S, Aurora SK, Recober A, Herial NA, Utley C, White L, Khuder SA. Childhood maltreatment and migraine (part I): Prevalence and adult revictimization, a multicenter headache clinic survey. Headache 2010; 50(1):20-31.

13. Oliveira PA, Scivoletto S, Cunha PZ. Estudos neuropsicológicos e de neuroimagem associados ao estresse emocional na infância e adolescência. Rev Psiquiatria Clínica 2010; 37(6):271-279.

14. Mesa-Gresa P, Moya-Albiol L. Neuro biology of child abuse: the "cycle of violence". Rev Neurol 2011; 52(8):489-503.

15. Mello MF, Faria AA, Mello AF, Carpenter LL, Tyka AR, Price LH. Maus-tratos na infância e psicopatologia no adulto: caminhos para a disfunção do eixo hipotálamo-pituitária-adrenal. Rev Brasileira de Psiquiatria 2009; 31(Supl. 2):S41-S48.

16. Tomoda A. Preliminary evidence for neurobiological and behavioral consequences of exposure to childhood maltreatment on regional brain development. No To Hattatsu 2011; 43(5):345-351.

17. Alink LRA, Cicchetti D, Kim J, Rogosch FA. Longitudinal associations among child maltreatment, social functioning, and cortisol regulation. Dev Psychol 2011; 48(1):224-236.

18. Harkness KL, Stewart JG, Wynne-Edwards KE. Cortisol reactivity to social stress in adolescents: role of depression severity and child maltreatment. Psychoneuroendocrinology 2011; 36(2):173-181.

19. Garbarino J. Psychological child maltreatment developmental view. Prim Care 1993; 20(2):307-315.

20. O'Hagan KP. Emotional and Psychological abuse: problems of definition. Child Abuse Negl 1995; 19(4):449-461.

21. Brassard MR, Hart DB. The psychological maltreatment rating scales. Child Abuse Negl 1993; 17(6):715-729.

22. Jellen LK, McCarroll JE, Thayer LE. Child emotional maltreatment: a 2-year study of US Army cases. Child Abuse Negl 2001; 25(5):623-639.

23. Claussen AH, Crittenden PM. Physical and psychological maltreatment relations among types of maltreatment. Child Abuse Negl 1991; 15(1-2):5-18.

24. Abranches CD, Assis SG. A (in)visibilidade da violência psicológica na infância e adolescência no contexto familiar. Cad Saude Publica 2011, 27(5):843-854.

25. Straus MA, Field CJ. Psychological aggression by American parents: national data on prevalence, chronicity and severity. Clin Child Fam Psychol Rev 2003; 65:795-808.

26. Elbedour S, Abu-Bader S, Onwuegbuzie AJ, Aref Abu-Rabia A, El-Aassam S. The scope of sexual, physical, and psychological abuse in a BedouinArab community of female adolescents: the interplay of racism, urbanization, polygamy, family honor, and the social marginalization of women. Child Abuse Negl 2006; 30(3):215-229.
27. Assis SG, Avanci JQ, Santos NC, Malaquias JV, Oliveira RV. Violência e representação social na adolescência no Brasil. Revista Panam Salud Publica 2004; 16(1):43-51.

28. Costa COM, Carvalho RC, Santa Bárbara JFR, Santos CAST, Gomes WA, Sousa HL. O perfil da violência contra crianças e adolescentes, segundo registros de Conselhos Tutelares: vítimas agressores e manifestações de violência. Cien Saude Colet 2007; 12(5):1129-1141.

29. Moura ATMS, Moraes CL, Reichenheim ME. Detecção de maus-tratos contra a criança: oportunidades perdidas em serviços de emergência na cidade do Rio de Janeiro, Brasil. Cad Saude Publica 2008; 24(12):2926-2936.

30. Rocha PCX, Moraes CL. Violência familiar contra a criança e perspectivas de intervenção do Programa Saúde da Família: a experiência do PMF/Niterói (RJ, Brasil). Cien Saude Colet 2011; 16(7):3285-3296.

31. Moeller TP, Bachmann GA, Moeller JR. The combined effects of physical, sexual, and emotional abuse during childhood: long-term health consequences for women. Child Abuse Negl 1993; 17(5): 623-640.

32. Spertus IL, Yehuda R, Wong CM, Halligan S, Seremetis SV. Childhood emotional abuse and neglect as predictors of psychological and physical symptoms in women presenting to a primary care practice. Child Abuse Negl 2003; 27(11):1247-1258.

33. Achenbach TM, Rescorla LA. Manual for the ASEBA School-age forms and profiles. Burlington: University of Vermont, Research Center for Children, Youth and Families; 2001.

34. Avanci JQ, Assis SG, Santos NC, Oliveira RVC. Escala de violência psicológica contra adolescentes. Rev Saude Publica 2005; 39(5):702-708.

35. Wagnild GM, Young HM. Development and psychometric evaluation of resilience scale. J Nurs Meas 1993; 1(2):165-178.

36. Pesce RP, Assis SG, Avanci JQ, Santos NC, Malaquias JV, Carvalhaes R. Adaptação transcultural, confiabilidade e validade da escala de resiliência. Cad Saude Publica 2005; 21(2):436-448.

37. Harding TW, De Arango MV, Baltazar J, Climent CE, Ibrahim HHA, Ladrido-Ignacio L, Murthy RS, Wig NN. Mental disorders in primary health care: a study of their frequency and diagnosis in four developing countries. Psychol Med 1980; 10(2):231-241.

38. Epstein NB, Baldwin LM, Bishop DS. The McMaster Family Assessment Device. Journal of Marital and Family Therapy 1983; 9:171-180.

39. Pitzner JK, Drummond PD. The Reliability and Validity of Empirically Scaled Measures of Psychological/Verbal control and Physical/Sexual Abuse: Relationship between current negative mood and a history of abuse independent of other negative life events. J Psychosom Res1997; 43(2);125-142.

40. Brasil.Conselho Nacional de Saúde (CNS). Resolução no 196/96, de Outubro de 1996. O Conselho Nacional de Saúde, no uso da competência que lhe é outorgada pelo Decreto no 93933 de 14 de janeiro de 1987, resolve. [documento da Internet]. 2013 [acessado 3 set 2013]. Disponível: http://conselho. saude.gov.br/resolucoes/reso_08.htm. 
41. Brasil. Ministério da Saúde (MS). Linha de cuidados para a atenção integral à saúde de crianças, adolescentes e suas famílias em situação de violências: orientação para gestores e profissionais de saúde. Ministério da Saúde, Secretaria de Atenção à Saúde. Departamento de Ações Programáticas Estratégicas. Brasília: MS; 2010.

42. Bagley C, Mallick K. Prediction of sexual, emotional and pshysical maltreatment and mental health outcomes in a longitudional cohort of 290 adolescent women. Child Maltreat 2000; 5(3):218-226.

43. Madu SN. The relationship between parental physical availability and child sexual, pshysical and emotional abuse: a study among a sample of university students in South Africa. Scand J Psychol 2003; 44(4):311-318.

44. Avanci JQ, Assis SG, Oliveira RVC. Sintomas depressivos na adolescência: estudo sobre fatores psicossociais em amostra de escolares de um município do Rio de Janeiro, Brasil. Cad Saude Publica 2008; 24(10):2334-2346.

45. Ravazolla MC. Resiliências Familiares. In: Melillo A, Ojeda ENS, organizadores. Resiliência: descobrindo as próprias fortalezas. Porto Alegre: Artmed; 2005. p. 73-85.

46. Moss E, Dubois-Comtois K, Cyr C, Tarabulsy GM, St-Laurent D, Bernier A. Efficacy of a home-visiting intervention aimed at improving maternal sensitivity, child attachment, and behavioral outcomes for maltreated children: a randomized control Trial. Dev Psychopathol 2011; 23(1):195-210.

Artigo apresentado em 17/03/2012

Aprovado em 23/04/2012

Versão final apresentada em 09/05/2012 